\title{
Automating Metadata for Dynamic Datasets
}

\author{
Jacqueline Mize \\ Radiance Technologies, Inc. \\ NOAA National Coastal Data Development Center \\ Building 1100, Room 101 \\ Stennis Space Center, MS 39529 \\ Dr. R. Ted Habermann \\ NOAA National Geophysical Data Center \\ 325 Broadway \\ Building DSRC, Room 1B152 \\ Boulder, CO 80305-3328
}

\begin{abstract}
Data and metadata are intrinsically connected. Both are vital components of a complete dataset and neither should be viewed as separate entities. Real-time and hourly data collections are dynamic, driving the need for amended metadata to record these types of observations.

Metadata is simply data about data that documents the characteristics of the data, such as condition, quality, and content. There is a vast array of various metadata standards such as the ISO 1911* series for Geographic information, the North American Profile of ISO (NAP), the Federal Geographic Data Committee (FGDC) standards, Directory Interchange Format (DIF) metadata, and library metadata standards such as Dublin Core (DC). Each of these standards is also available in a variety of formats, such as text, HTML, XML, and FAQ views.

Creating and maintaining various types of metadata for dynamic data can prove to be a daunting task. Automating metadata creation and maintenance processes is an approach to combat the possible resource drain that can be caused by constantly evolving metadata, especially if that metadata is required to be output in a variety of formats and standards.

Metadata can be automated by collaboratively using a variety of input sources such as multiple databases, reports, and active sensors to create and maintain dynamic metadata in a variety of formats and standards through the use of eXtensible Stylesheet Transforms (XSLTs) and eXtensible Stylesheet (XSLs). Directly creating various metadata formats from the input sources, rather than translating between various standards, aids in preserving information. In legacy data cases, XSLTs can be used to translate between metadata standards. The transformation process could be triggered by updating or altering the input data sources. Various trans-forms and/or stylesheets would be selected, depending on the trigger, applied to the altered input, and result in the generation of multiple records in a variety of standards and formats.
\end{abstract}

\section{INTRODUCTION}

\section{A. Multiple Metadata Standards}

Metadata is the standardized documentation of data. The issue is that this "standardized documentation" is available in an assortment of standards. As the number of metadata standards continues to grow, the question arises, "Why not have a common standard?" Often, discipline-specific communities develop standards specifically to cater to explicit data types, structures, and procedures, and even tailoring to suit a core set of users [1]. Metadata is not as simple as "one size fits all.". No single standard can address all classes of metadata, i.e., descriptive, operational, and interface-specific metadata [2]. It is also unrealistic to constrain domain-specific users to generalized standards that cannot satisfy the needs of their respective user community.

Exchanging information across these standards and facilitating interoperability requires an understanding of multiple metadata standards. Often, these various standards are complex and difficult to fully understand, with conventional metadata management necessitating the staffing of experienced personnel. Training personnel to be well-versed in various metadata standards (i.e., FGDC Standard for Digital Geospatial Metadata (CSDGM), International Organization for Standardization (ISO) 191** series metadata, Directory Interchange Format (DIF), and Ecological Metadata Language (EML), just to name a few,) consumes much time and many resources. Numerous metadata standards overlap and intersect [3]. Metadata crosswalks enable the conversion from one standard to another. Take, for example, crosswalks.

Crosswalks define "how" to get metadata from one standard into another. They are often tables, charts, or spreadsheets that identify the equivalencies, gaps, and relationships between metadata standards [1]. Crosswalks can also be used when metadata is unavailable. Often, the data needed to create metadata is stored in databases. Databases have a defined structure or schema. 
Relationships are defined and enumerations set. These database structures can be used to crosswalk into a standard, in the same fashion as if one were creating a crosswalk between two existing metadata standards.

B. Multiple Metadata Formats

Metadata is not only available in various standards, but also in multiple formats. There are various views of metadata such standards as text, HyperText Markup Language (HTML), and Extensible Markup Language (XML) (Fig. 1). Text views are simple and, easily read, but are more of a legacy type of view. Text documents can be easily corrupted, but. XML views preserve the information; however, they are mainly for machine-to-machine applications. HTML views display the XML in an easy- toread manner. HTML views are often customized for particular applications and are often user-specific.

\section{Flexibility of XML}

XML defines a set of rules for defining a documents content, structure, and behavior. It is a very flexible language and easily adapted to technological changes. XML is extremely functional for a variety of applications, especially when trying to exchange data amid heterogeneous databases [4]. Web content systems, which are XML-based, enable the integration of information to constituents in real time [5]. As a subset of the Standard Generalized Markup Language (SGML), XML was originally designed to ease implementation and to allow interoperability with SGML and HyperText Markup Language (HTML) [6]. XML facilitates usability by allowing applications to dynamically discover, access, view, and share information across different structured metadata standards.

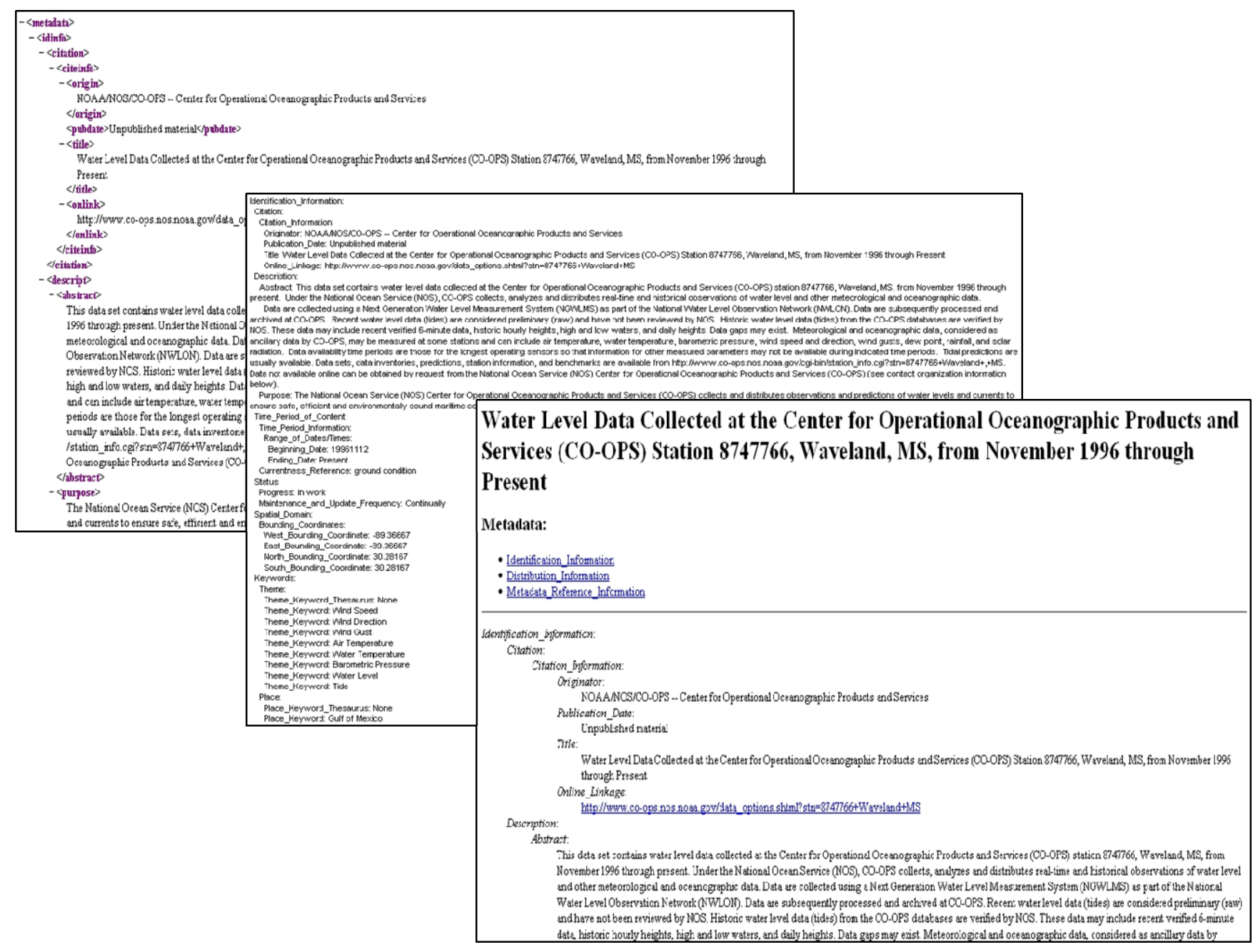

Fig. 1 XML, text, and HTML formats of the same metadata record. 


\section{XSLT and XSL}

Metadata can be automated by collaboratively using a variety of input sources, such as multiple databases, reports, and active sensors, to create and maintain dynamic metadata in a variety of formats and standards through the use of eXtensible Stylesheet Transforms (XSLTs) and eXtensible Stylesheets (XSLs). XSLTs perform the translations between standards and are used to apply what is defined by crosswalks. An XSLT can be applied to an XML document of one metadata standard to produce an XML document of another standard; XSLTs can be used to transform a variety of sources, such as a database and spreadsheet, to result in XML documents in the targeted structure [7].

XSLs provide instruction and define how the information from the XML is displayed and viewed. These views can be customized for various applications or user preferences to produce various presentations of the information. Applying stylesheets to an XML document produces the desired metadata views, such as text, HTML, and other customized views. The use of XSLs allow maximum interoperability by allowing the content to be created once, as XML and, with the use of multiple XSLs, can produce infinite formats and views of the information.

\section{Automating MetadATA}

\section{A. XML and Dynamic Data}

Collection-level metadata may be static, that is, documenting general details about a collection. But a majority of data being collected is not static; therefore, accurate granular metadata should not be static as well. Collection-level metadata identifies and documents static information. The collection level metadata is often used as a template to build the granular metadata for the data. Once static information is established for the collection, dynamic information fills the voids to create granular metadata. Updating or altering the input data sources could trigger the transformation processes, selecting various transforms and/or stylesheets to be applied to the altered input and generate multiple metadata records in a variety of standards and formats, depending on the processes triggered.

Many data formats, such as netCDF, are self-documenting and can be used to populate the metadata. In other cases, dynamic information, such as coverage and instrumentation details, can then be pulled from the data itself and added to the template to produce the granular metadata. This can often be accomplished in real-time from the result of a trigger or created on a scheduled basis to obtain dynamically driven metadata.

\section{B. Automating Metadata Creation}

There are many methods to using the cost-effective approach of automating metadata. Using XML technologies to directly create various metadata standards and formats from the input sources, rather than by translating between various standards, aids in preserving information and is the ideal method. Existing capabilities, as well as legacy collections, can be enhanced if access to the source is not feasible [7].

\section{Automating Metadata Standards and Formats}

The NOAA Metadata Manager and Repository (NMMR) is a system for archiving and publishing metadata that uses OracleXML as the back end [8]. The Oracle XML data base leverages the flexibility of XML technologies (Oracle). The NMMR fosters interoperability from taking the XML information and providing metadata in FGDC, ISO, DIF, NetCDF Markup Language (NcML), and Thematic Real-time Environmental Distributed Data Services (THREDDS) standards by using various XSLTs (Fig. 2). A variety of formats (views) created from XSLs are also provided: HTML, custom FAQ view, Text, XML, Keyhole Markup Language (KML), and various other custom views, such as table formats and a rubric view that assesses the content (Fig. 3).

\section{PROPOSED METHODS}

\section{A. From the Source}

Metadata can instantly be derived directly from the source. Static information could be detailed ahead of collection time while dynamic information triggers the creation of granular metadata. NOAA's Shipboard Computer System (SCS) [9 ], for example, could be used to create such metadata. The SCS gathers, processes, and disseminates data from the NOAA Ocean Explorer. Many of the ship's instruments are also connected to the SCS. Continuous, or "underway," data is logged, as well as eventtriggered data. If the SCS populated a database that could leverage XML with the dynamic information being collected, XSLTs and XSLs could be applied to produce granular metadata in multiple metadata standards in a variety of formats almost instantly as seen in the sample workflow (Fig. 4). 


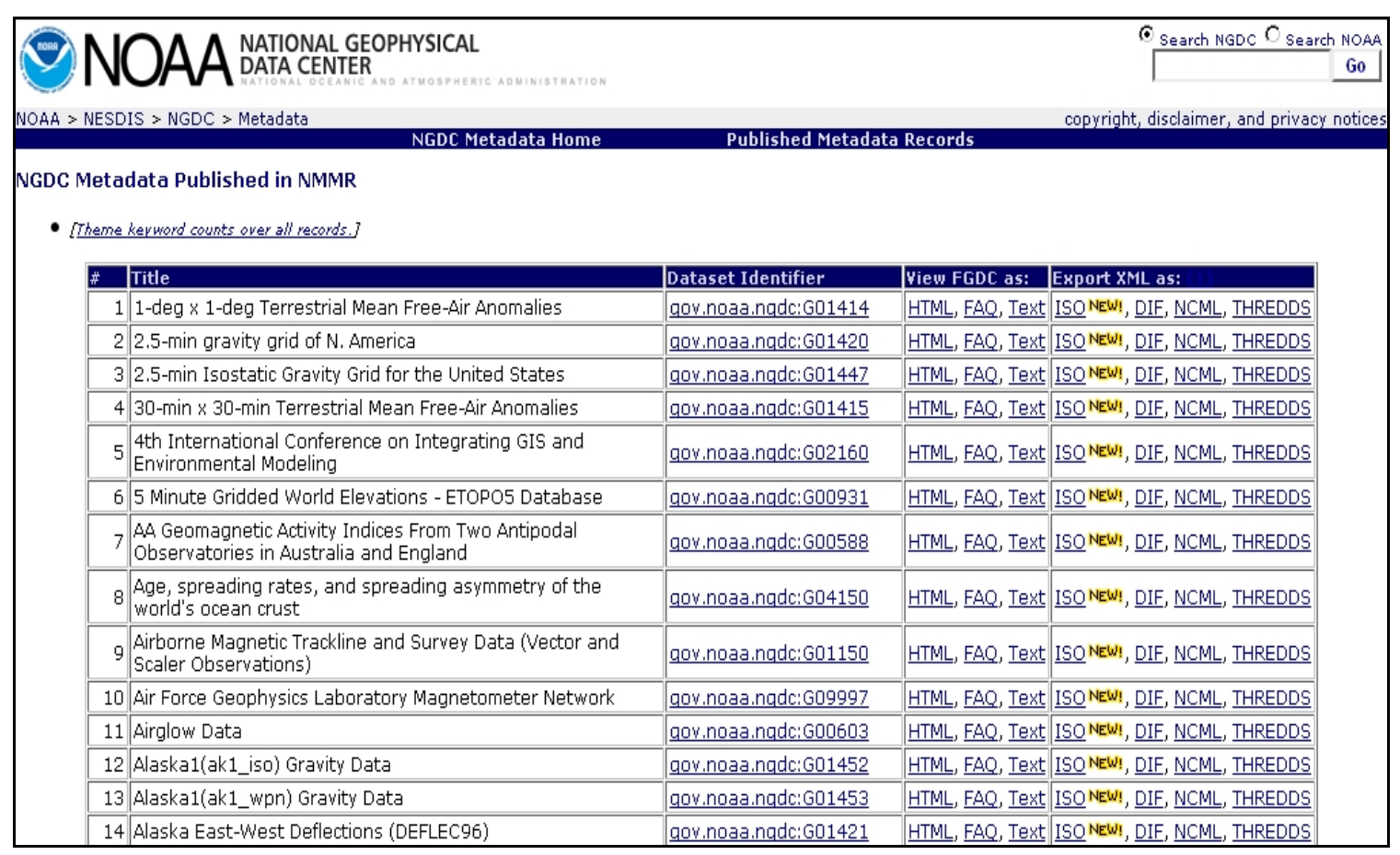

Fig. 2 NGDC offers several different Metadata Standards.

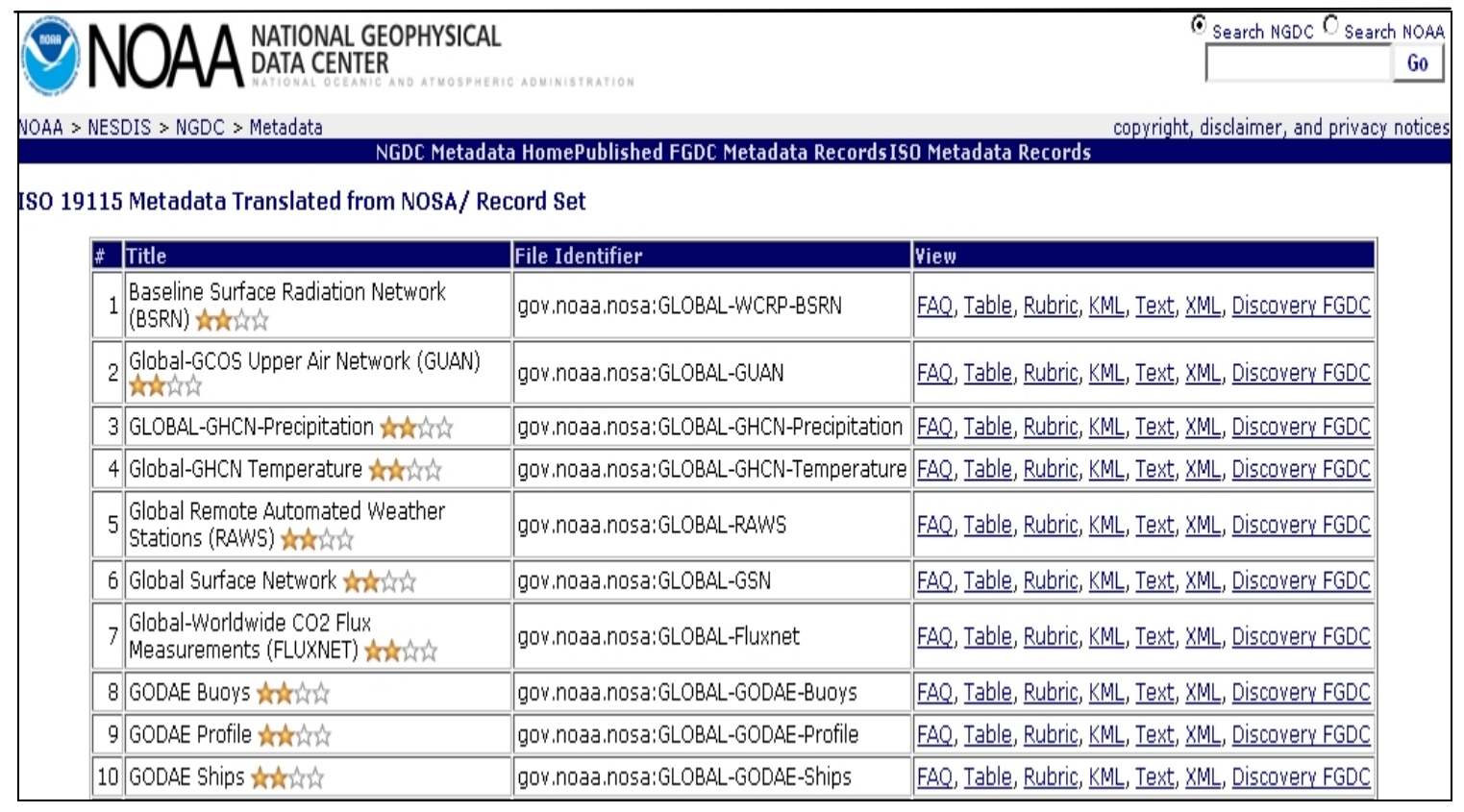

Fig. 3 ISO Metadata available in a variety of formats. 


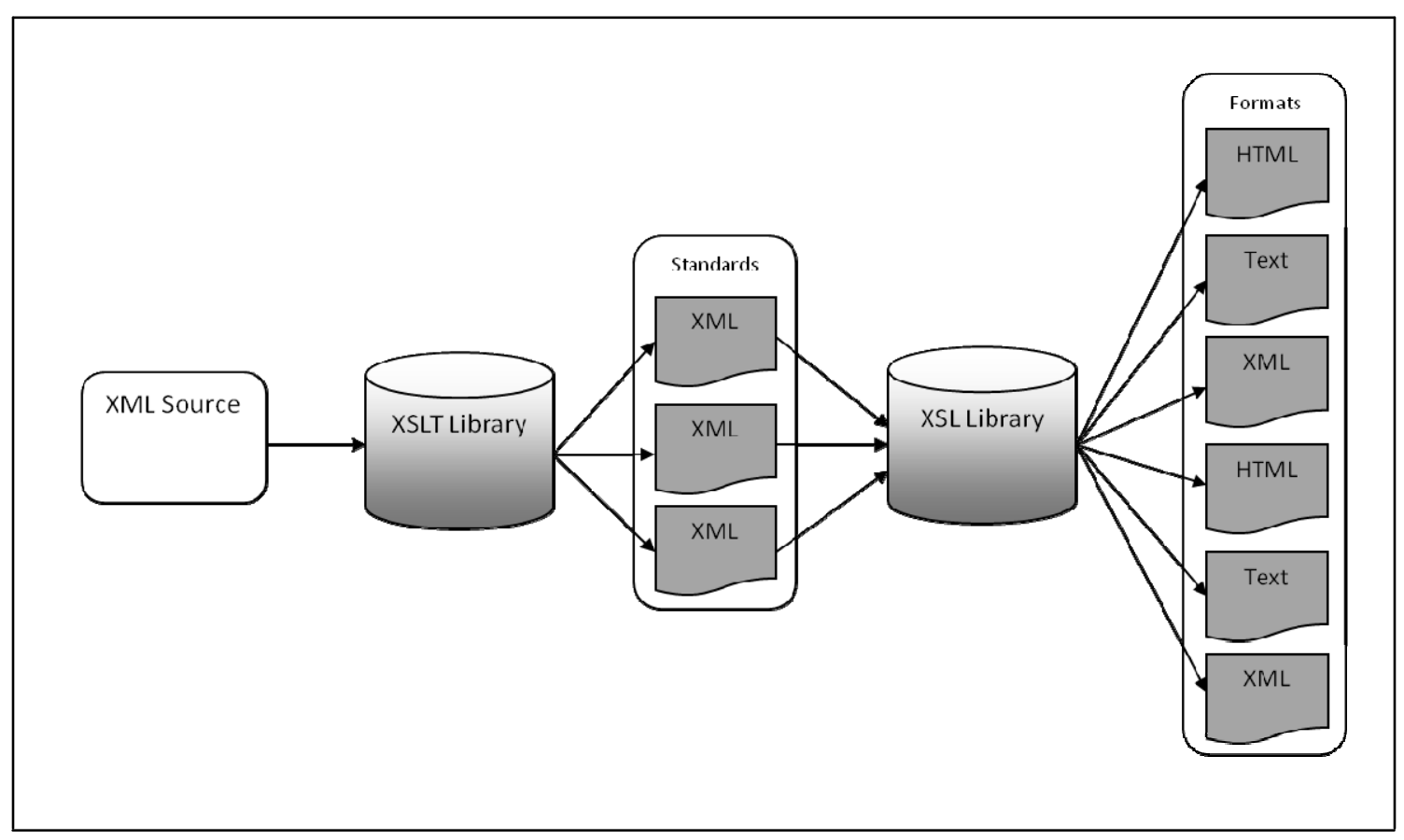

Fig. 4 Workflow

\section{B. Legacy}

If direct access from the source is not feasible, interoperability can still be accomplished with a similar workflow (Fig. 4). In legacy data and metadata cases, XSLTs can be used to translate between metadata standards. The resulting XML documents-in various standards-may then have various stylesheets applied to produce custom formats of the metadata.

\section{CONCLUSIONS}

Creating and maintaining various types of metadata for dynamic data is a daunting task, but it can be combatted through the automation of metadata. With the use of XSLTs and XSLs, it is now feasible to provide accurate documentation of real-time data of multiple metadata standards and various views of these standards to maximize interoperability and to increase efficiency.

Ideally, metadata will be created directly from the input sources. These new technologies have enabled the automatic creation of granular metadata directly from dynamic data, aiding in the preservation, archival, and dissemination of data in a timely manner. In cases where the input source is not available, legacy metadata could be used. Directly creating various metadata formats from the input sources, rather than translating between various standards, also aids in preserving information. Maintaining multiple standards and formats will also be more efficient. The source XML is the only piece that needs to be maintained, providing a single editing point. If updates are made to the source XML, this event could serve to trigger the automated process to create the desired metadata standard and format outputs. The output files can then be disseminated and accessed through a multitude of applications, thus increasing the interoperability and discoverability with minimum impact to the resource.

\section{REFERENCES}

[1] Lightle, Kimberly, and Judith Ridgway. "Generation of XML Records across Multiple Metadata Standards.” D-Lib Magazine 9.9 (2003): n. pag. Web. 22 Jun 2010. <http://www.dlib.org/dlib/september03/lightle/09lightle.html>.

[2] "Metadata Architecture and Standards." Sandpiper Software. Sandpiper Software, n.d. Web. 25 Jun 2010. <http:// www.sandsoft.com/metadata.html $>$.

[3] Harpring, Patricia. "Metadata Standards Crosswalk." The Getty. J. Paul Getty Trust, 09 Jun 2009. Web. 25 Jun 2010. <http:// www.getty.edu/research/conducting_research/standards/intrometadata/crosswalks.html $>$. 
[4] December, John. "XML: Extensible Markup Language.” December Communications, Inc.. December Communications, Inc., 12 May 2010. Web. 22 Jun 2010. <http://www.december.com/html/tech/xml.html $>$.

[5] Vogel, Jeffrey. "How XML enables dynamic content." Network World 13 Mar 2000: n. pag. Web. 22 Jun $2010 .<$ http:// www.networkworld.com/news/tech/0313tech.html>.

[6] Bray, Tim, Jean Paoli, C.M. Sperberg-McQueen, Eve Maler, and Francois Yergeau. "Extensible Markup Language (XML) 1.0 (Fifth Edition)." World Wide Web Consortium (W3C). N.p., 26 Nov 2008. Web. 14 Jul 2010. <http://www.w3.org/ TR/2008/REC-xml-20081126/>.

[7] Mize, Jacqueline, and Christopher Robertson. "A Solution to Metadata: Using XML Transformation to Automate Metadata." Oceans 2009. Biloxi, MTS/IEEE. 2009. Web. 14 Jul 2010.

[8] “NMMR For Dummies.” NOAA National Geophysical Data Center (NGDC). NGDC, n.d. Web. 14 July $2010 .<$ http://ngdc. noaa.gov/nmmr/usersGuide/NmmrForDummies.html>.

[9] Shipboard Computer System (SCS). NOAA Ocean Explorer. N.p., 24 Feb 2006. Web. 19 Jul 2010. <http://oceanexplorer. noaa.gov/technology/tools/scs/scs.html>. 